\title{
XII. THE OSMOTIC PRESSURE OF HAEMOGLOBIN.
}

\section{AN EXPLANATION OF THE EFFECT OF ACETIC ACID, BASED ON DONNAN'S THEORY OF MEMBRANE EQUILIBRIUM.}

\author{
BY HELENE CONNET WILSON. \\ From the Department of Physiological Chemistry, School of Medicine, \\ University of Pennsylvania.
}

(Received December 23rd, 1924.)

IN a recent publication [Wilson, 1923] on "The Effect of Carbon Dioxide and Acetic Acid on the Osmotic Pressure of Haemoglobin," it was shown that "the osmotic pressure of solutions of purified haemoglobin is three or four times as great when dialysed against acetic acid or water saturated with $\mathrm{CO}_{2}$ (both about $p_{\mathrm{H}} 4$ ) as against water alone." Reasons were given for believing that "this is due to the formation of some sort of ionising salt of haemoglobin, either one which ionises into several protein ions, or into protein and acetate or protein and bicarbonate ions" (p. 70). It was pointed out that a study of the hydrogen-ion concentration of the haemoglobin solution inside the osmometer and the fluid outside the osmometer should determine which kind of dissociation had taken place. If the ionisation were into protein ions only, the Donnan equilibrium [1911] should not come into play and the hydrogen-ion concentration of the inner and outer solutions in the osmometer should be the same when equilibrium was reached. If a haemoglobin acetate were formed which dissociated into haemoglobin and acetate ions, then the Donnan equilibrium would, presumably, occur and the inner solution would be less acid than the outer. The latter hypothesis is substantiated by the experiments to be reported in this paper. The conclusion is, therefore, drawn that the increased osmotic pressure observed is due to the formation of haemoglobin acetate. The resulting equilibrium relations have been quantitatively consídered.

\section{Experimental.}

The haemoglobin ${ }^{1}$ was several times recrystallised according to the method of Dudley and Evans [1921], which depends on the fact that reduced haemo-

1 The haemoglobin used in these experiments was prepared from the corpuscles of oxalated horse blood, obtained through the kindness of the Mulford Biological Laboratories. 
globin is much more soluble than oxyhaemoglobin. The osmometers consisted of collodion sacs attached to mercury manometers. The method of preparing the purified haemoglobin and the description of the osmometers are fully given in the previous communication [Wilson, 1923]. In the present experiments larger osmometers (holding about 15 cc. haemoglobin) were used in order to have enough haemoglobin solution for duplicate determinations of the hydrogen-ion concentration.

A typical experiment was carried out as follows. Several collodion sacs were filled with a freshly prepared 1 to $2 \%$ solution of crystalline oxyhaemoglobin and dialysed against distilled water previously boiled and saturated with oxygen. The osmometer flask was loosely stoppered, so that sufficient carbon dioxide entered to make the outer fluid somewhat more acid than $p_{\mathrm{H}} 7 \cdot 0$. Most of the osmometers were kept in the ice box at about $2^{\circ}$. The outer fluid was renewed several times daily until the reaction remained constant and the osmotic pressure of the haemoglobin solution did not change. The osmotic pressure of a $1 \%$ solution was about $10 \mathrm{~mm}$. $\mathrm{Hg}$, which agrees quite well with the calculated pressure using 16,600, the commonly accepted molecular weight of haemoglobin, as determined by several methods [Gamgee, 1898; Hüfner and Gansser, 1907; Barcroft and Hill, 1910].

Replacing the distilled water with weak acetic acid of $p_{\mathrm{H}} 3 \cdot 8$ to $4 \cdot 0$ (about $0.001 M$ ) resulted in a four- to six-fold increase in the osmotic pressure. The acetic acid was renewed once or twice a day until the reaction of the outer fluid (tested colorimetrically) remained constant (one to two days). The elimination of the inner core of liquid in the osmometer by means of a glass float permitted the system to come to equilibrium rapidly. To be certain that equilibrium was obtained, however, most of the osmometers were allowed to stand for 3 or 4 days. The reactions of the haemoglobin solution and the acetic acid outer fluid were then determined by means of the hydrogen electrode. As a check on the permeability of the sacs to acetic acid, a sac containing distilled water $\left(p_{\mathrm{H}} 6 \cdot 0-7 \cdot 0\right)$ was dialysed against acetic acid of $p_{\text {H }} 4 \cdot 0$. The $p_{\text {H }}$ of the fluid inside the sac became $4 \cdot 0$ in less than 24 hours.

At the end of an experiment the haemoglobin from one or two of the osmometers was filtered and the percentage haemoglobin determined by evaporating a sample to dryness. It was always found that during the experiment some of the haemoglobin had been precipitated out of the solution, the reduction in concentration being $15 \%$ or less. With the weak solutions used, this decrease in concentration introduced only a slight error in the comparison of osmotic pressure readings at the beginning and end of an experiment.

No attempt was made to determine the amount of conversion of oxyhaemoglobin into reduced haemoglobin and methaemoglobin. In the previous paper several reasons were given for believing that, under the conditions of these experiments, splitting of the haemoglobin molecule did not occur.

Typical results of the acetic acid experiments in four osmometers (two different samples of oxyhaemoglobin being used) are as follows:

Bioch. xIx 


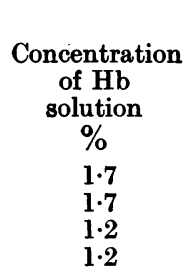

\begin{tabular}{|c|c|}
\hline Osmotic pres & of $\mathrm{Hb}$ solut \\
\hline $\begin{array}{l}\text { Against } \\
\text { water } \\
\text { mm. Hg }\end{array}$ & $\begin{array}{l}\text { Against } \\
\text { acetic acid } \\
\text { mm. Hg }\end{array}$ \\
\hline 16 & 70 \\
\hline 15 & 71 \\
\hline 10 & 50 \\
\hline 8 & 50 \\
\hline
\end{tabular}
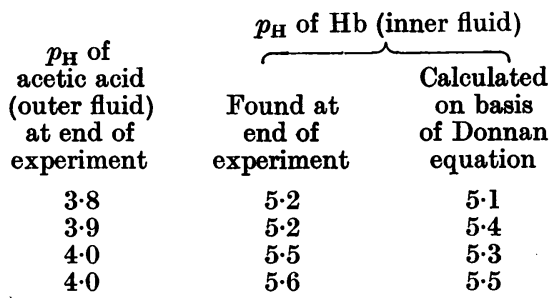

It will be seen from an examination of the fourth and fifth columns of the table that, at the end of such an experiment as has been described, the haemoglobin solution was considerably less acid than the acetic acid outer fluid, at a time when the osmotic pressure had increased four- to six-fold. This increased pressure might conceivably be due to one of a number of different causes, but, as was shown in the previous paper [Wilson, 1923], in every case, except that of the formation of haemoglobin acetate, the acetic acid would distribute itself so as to give the same hydrogen-ion concentration on each side of the membrane. In the case of the formation of haemoglobin acetate, however, the acetate ions therefrom would prevent an equal distribution of ions from the acetic acid, in accordance with Donnan's theory of membrane equilibrium. When the data are studied quantitatively, using the Donnan equation, it is found that the haemoglobin solution inside the osmometer should be less acid than the outer fluid, as was experimentally found to be the case. The increased osmotic pressure would be due to the haemoglobin ions and the acetate ions of the haemoglobin acetate (three to five acetate ions being necessary to account for the four- to six-fold increase in osmotic pressure). The counter osmotic pressure in these experiments is too slight to influence the calculations.

The calculations are necessarily rough, due to certain shortcomings in the data. The osmotic pressure readings are not quite as uniform as one would wish and the concentration of the haemoglobin undergoes a certain diminution during the course of the experiment. In postulating three to five acetate radicles for each haemoglobin radicle to account for the osmotic pressure change, it is assumed that there is no aggregation of the haemoglobin. If there were aggregation the ratio of acetate radicles to haemoglobin radicles would have to be larger.

Despite these sources of error, columns 5 and 6 in the table show that there is a relatively close agreement between the calculated and experimental values.

The calculations for the first of the experiments listed in the table above are given herewith.

Letting $R$ be the cation of haemoglobin acetate, (1) outer fluid, (2) inner fluid, and $x, y$ and $z$ ion concentrations, we have the following scheme when equilibrium in the osmometer is attained. 
(2)

(R) $\quad\left(\mathrm{CH}_{3} \mathrm{CO}_{2}\right) z$

\begin{tabular}{cccc}
$z$ & $\left(\mathrm{CH}_{3} \mathrm{CO}_{2}\right) z$ & \\
& $\left(\mathrm{CH}_{3} \mathrm{CO}_{2}\right) z$ & $(\mathrm{H})$ & $\left(\mathrm{CH}_{3} \mathrm{CO}_{2}\right)$ \\
$(\mathrm{H})$ & $\left(\mathrm{CH}_{3} \mathrm{CO}_{2}\right)$ & $x$ & $x$ \\
$y$ & $y$ & & \\
& $\left(\mathrm{CH}_{3} \mathrm{CO}_{2} \mathrm{H}\right)=\left(\mathrm{CH}_{3} \mathrm{CO}_{2} \mathrm{H}\right)$ \\
& \multicolumn{3}{c}{$y(y+3 z)=x^{2}}$.
\end{tabular}

When the $p_{\mathrm{H}}$ of the outer fluid is $3 \cdot 8, x=0.00016 \mathrm{~N}$. A $1.7 \%$ haemoglobin solution (molecular weight of haemoglobin 16,600 ) is $0.0010 M$, hence

Substituting in

$$
\begin{aligned}
& z=0.0010 M . \\
& y(y+3 z)=x^{2}, \\
& y(y+0.003)=0.0000000256, \\
& y^{2}+0.003 y=0.0000000256 .
\end{aligned}
$$

$y^{2}$, being infinitesimal as compared with the other values, may be disregarded. Hence

$$
y=0.0000085 \text {. }
$$

The hydrogen-ion concentration $(y)$ of the inner fluid which contains the haemoglobin acetate is, therefore, $0 \cdot 0000085 N\left(p_{\mathrm{H}}=5 \cdot 1\right)$, while the hydrogenion concentration $(x)$ of the outer fluid which contains only acetic acid is $0.00016 N\left(p_{\mathrm{H}}=3 \cdot 8\right)$. The experimental value, as seen in the table, is $5 \cdot 2$, instead of the calculated $5 \cdot 1$.

The results strongly indicate that the Donnan equilibrium was operating in these experiments and that haemoglobin acetate is formed when acetic acid is used as outer fluid in an osmometer with haemoglobin on the inside.

This conclusion can doubtless be extended to the formation of other acid salts of haemoglobin, under similar conditions, the hydrogen-ion concentration apparently being the determining factor. In the previous paper carbonic and lactic acids of the same $p_{\mathrm{H}}$ as the acetic acid used in these experiments gave identical osmotic pressure results.

\section{SUMMARY.}

When haemoglobin is dialysed in an osmometer against acetic acid, there results a four- to six-fold increase in osmotic pressure above the value against distilled water. When equilibrium is reached the hydrogen-ion concentration of the haemoglobin solution is found to be considerably less than that of the acetic acid outer fluid. Such an unequal distribution of hydrogen ions may be explained by Donnan's theory of membrane equilibrium. The existence of a Donnan equilibrium under these conditions proves that haemoglobin acetate has been formed. The formation of haemoglobin acetate adequately explains the increased osmotic pressure.

\section{REFERENCES.}

Barcroft and Hill (1910). J. Physiol. 39, 411.

Donnan (1911). Z. Electrochem. 17, 572.

Dudley and Evans (1921). Biochem. J. 15, 487.

Gamgee (1898). "Haemoglobin," in Schäfer's Textbook of Physiology, 1, 185.

Hüfner and Gansser (1907). Arch. Anat. Physiol. 299.

Wilson (1923). Biochem. J. 17, 59. 